\title{
Ischemic Bowel Disease
}

National Cancer Institute

\section{Source}

National Cancer Institute. Ischemic Bowel Disease. NCI Thesaurus. Code C35212.

Disease of the large or small intestine that is caused by inadequate blood supply. 